\title{
MicroRNA miR-9 Modifies Motor Neuron Columns by a Tuning Regulation of FoxP1 Levels in Developing Spinal Cords
}

\author{
Gaizka Otaegi, ${ }^{1}$ Andrew Pollock, ${ }^{1,2}$ Janet Hong, ${ }^{1}$ and Tao Sun ${ }^{1,2}$ \\ ${ }^{1}$ Department of Cell and Developmental Biology, Weill Medical College of Cornell University and ${ }^{2}$ Weill Cornell Graduate School of Medical Sciences, New \\ York, New York 10065
}

\begin{abstract}
The precise organization of motor neuron subtypes in a columnar pattern in developing spinal cords is controlled by cross-interactions of multiple transcription factors and segmental expressions of Hox genes and their accessory proteins. Accurate expression levels and domains of these regulators are essential for organizing spinal motor neuron columns and axonal projections to target muscles. Here, we show that microRNA miR-9 is transiently expressed in a motor neuron subtype and displays overlapping expression with its target gene FoxP1. Overexpression or knockdown of miR-9 alters motor neuron subtypes, switches columnar identities, and changes axonal innervations in developing chick spinal cords. miR-9 modifies spinal columnar organization by specifically regulating FoxP1 protein levels, which in turn determine distinct motor neuron subtypes. Our findings demonstrate that miR-9 is an essential regulator of motor neuron specification and columnar formation. Moreover, the overlapping expression of miR-9 and its target FoxP1 further illuminates the importance of fine-tuning regulation by microRNAs in motor neuron development.
\end{abstract}

\section{Introduction}

In developing spinal cords, motor neurons (MNs) and interneurons (INs), which are specified from progenitor cells, construct accurate connections to target muscles and other neurons (Jessell, 2000; Dasen, 2009; Goulding, 2009). The spinal MNs are organized into distinct columns that project axons to different peripheral regions along the rostrocaudal axis (Dasen, 2009). Median motor column (MMC) neurons innervate axial muscles, hypaxial motor column (HMC) neurons innervate body wall muscles around the rib cage, preganglionic motor column (PGC) neurons innervate sympathetic ganglia, and lateral motor columns (LMC) innervate limb muscles (Fetcho, 1992; Gutman et al., 1993; Landmesser, 2001). LMC neurons occur only in the brachial and lumbar regions in spinal cords, which are aligned with the forelimbs and hindlimbs, respectively, in mammals and wings and legs in birds. In the chick, preganglionic autonomic motor neurons, termed Column of Terni (CT) neurons, are positioned in the dorsomedial spinal cord at thoracic levels and

Received Aug. 18, 2010; revised Sept. 29, 2010; accepted 0ct. 18, 2010.

This work was supported in part by a postdoctoral fellowship from the Basque Government (G.0.), the Whitehall Foundation (T.S.), the Ellison Medical Foundation (T.S.), and National Institutes of Health/National Institute of Mental Health Grant R01-MH083680 (T.S.). We thank members of the Sun laboratory for their valuable discussions and advice. We thank Tom Jessell for anti-Is 1 /2 antibodies, Ben Novitch for anti-FoxP1 antibodies, Connie Cepko for anti-Chx10 antibodies, and Susan Morton, Dan Vasiliauskas, and Ed Laufer for anti-pSmad antibodies.

G.O. and T.S. designed research. G.O., A.P., and J.H. performed research. G.O. and T.S. analyzed data and wrote the manuscript.

Correspondence should be addressed to Tao Sun, Department of Cell and Developmental Biology, Cornell University Weill Medical College, 1300 York Avenue, Box 60, Room W820A, New York, NY 10021. E-mail: tas2009@med.cornell.edu.

DOI:10.1523/JNEUROSCI.4330-10.2011

Copyright $\odot 2011$ the authors $\quad 0270-6474 / 11 / 310809-10 \$ 15.00 / 0$ project axons to sympathetic targets (Prasad and Hollyday, 1991; Gutman et al., 1993; Cornbrooks et al., 1997).

Spinal motor neurons are organized in columns in a segmental manner, which is controlled by Hox proteins and cofactors (Dasen et al., 2003, 2005; Shah et al., 2004; Wu et al., 2008; Jung et al., 2010). A Forkhead domain transcription factor FoxP1 displays high expression in LMC MNs at the brachial and lumbar levels but low expression in PGC MNs at the thoracic level. Altered FoxP1 levels switch MN columnar organization and axonal projections, implying the importance of expression dosage and domains of FoxP1 in MNs (Dasen et al., 2008; Rousso et al., 2008). However, the mechanisms behind FoxP1 expression level regulation in MNs remain unclear.

The discovery of microRNAs (miRNAs) has revealed a new mechanism of gene regulation during development. miRNAs are $\sim 22$ nt endogenous noncoding small RNAs (Lee et al., 1993; Wightman et al., 1993). A mature miRNA recognizes the 3' untranslated region (UTR) of its target mRNA and affects mRNA stability and/or silences protein translation (Carthew and Sontheimer, 2009; Kim et al., 2009). Because miRNAs silence target proteins, it is likely that an miRNA and its target display nonoverlapping expression (Bartel and Chen, 2004; Hornstein et al., 2005; Stark et al., 2005). However, recent work has shown overlapping expression of miRNAs and target mRNAs in the same cells (Karres et al., 2007). It appears that miRNAs function by optimizing protein output in cells, which indicates miRNA as fine-tuned regulators of the expression levels of their targets (Hobert, 2007; Karres et al., 2007; Biryukova et al., 2009; Shkumatava et al., 2009).

Here, we show that miR-9 is expressed in MNs in the LMC, overlapping with FoxP1, but not in the MMC of developing chick 

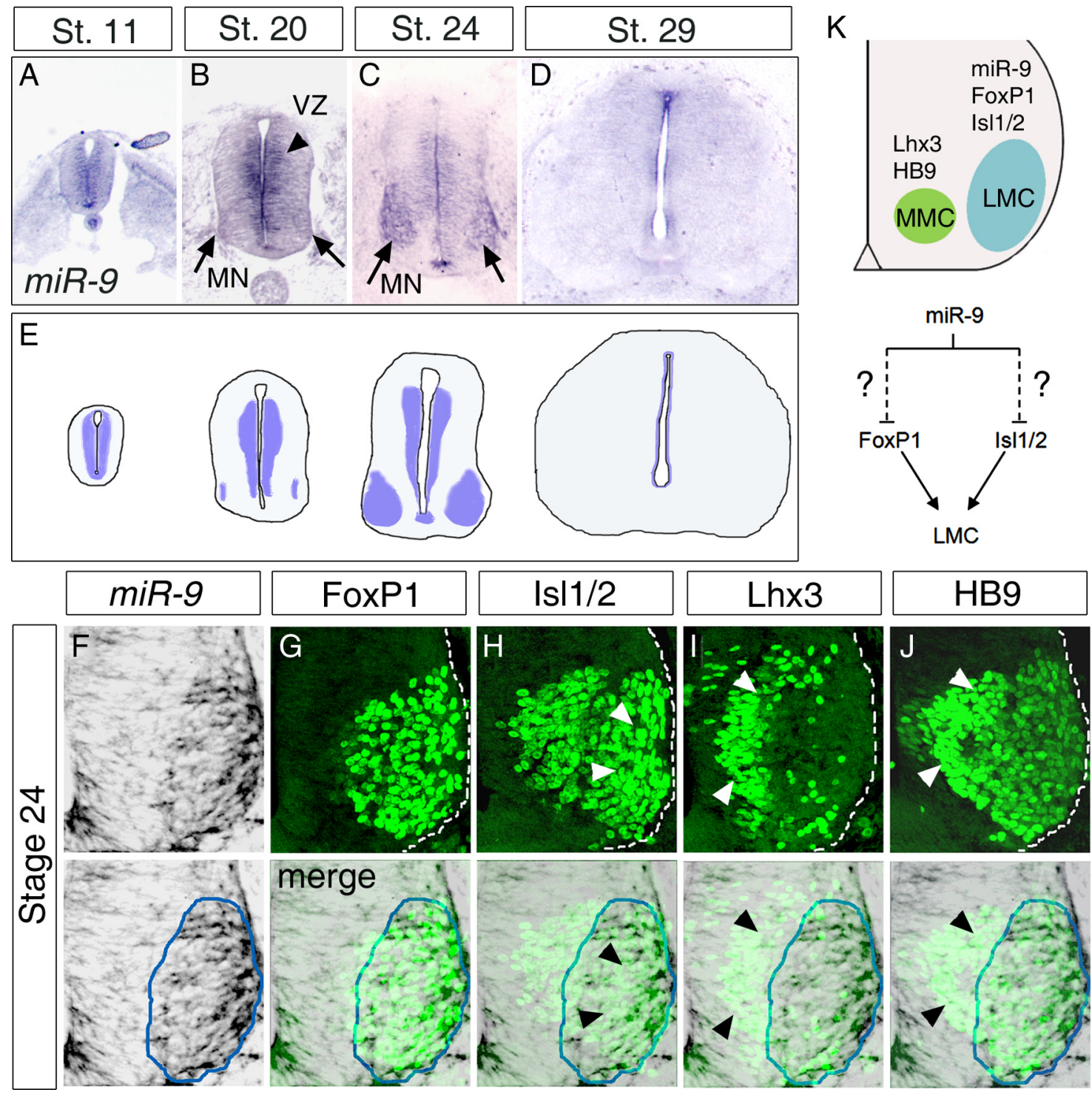

Figure 1. Dynamic expression of miR-9 in chick developing spinal cords. A-D, miR-9 expression patterns in chick spinal cords from stage (St.) 11 to stage 29 , as detected by in situ hybridization. miR-9 was highly expressed in the VZ (arrowhead) and transiently in MNs (arrows). $\boldsymbol{E}$, A schematic summary of miR-9 expression. $\boldsymbol{F}$, High-power view of miR-9 expression in MNs (highlighted) in the ventral horn of stage 24 chick spinal cords. G-J, Merged images of miR-9 expression with motor neuron markers FoxP1, Is11/2, Lhx3, and HB9 in MNs. Higher expression of Lhx3 and HB9 in the MMC is labeled (arrowheads). $\boldsymbol{K}$, Summary of miR-9 expression in motor neuron columns. miR-9 is expressed in MNs in the LMC in which FoxP1 and Is $11 / 2$ are expressed but not in MNs in the MMC in which Lhx3 and HB9 are expressed. miR-9 may negatively regulate FoxP1 and/or IsI1/2 expression levels and define the LMC.

spinal cords. Overexpression of miR-9 switches LMC into MMC and subsequently alters $\mathrm{MN}$ axonal projections to their targets. miR-9 functions through regulating expression levels of its target protein FoxP1 in LMC and in CT neurons. Our findings reveal a fine-tuning regulation of miR-9 in modifying $\mathrm{MN}$ columnar organization in the spinal cord.

\section{Materials and Methods}

miR-9 expression constructs. The $88 \mathrm{nt}$ precursor hairpin sequences of miR-9-1 and miR-9-2 and $100 \mathrm{nt}$ of genomic sequences flanking each side of the hairpin sequence were amplified by PCR from the genomic loci of chick miR-9-1 and miR-9-2. The following primers were used: chick miR-9-1 amplification primers, forward, 5' -ctcacggtacggggatgg- $3^{\prime}$ and reverse, 5' -gacaaaaccctgtgcagtg- $3^{\prime}$; chick miR-9-2 amplification primers, forward, $5^{\prime}$-gctcctggaggagacagc- $3^{\prime}$ and reverse, $5^{\prime}$-gctgtaaaagcgttcggtg- $3^{\prime}$.

To generate miR-9 expression constructs, the $290 \mathrm{nt}$ DNA fragment was inserted into an expression vector pCAGIG for in ovo electroporation and in pcDNA3.1 for transfection in cell lines. The pCAGIG vector contains a reporter gene green fluorescent protein (GFP), which allows us to trace the electroporated cells. For the control expression construct, the wild-type miR-9-1 seed sequence $5^{\prime}$-CUUUGGU- ${ }^{\prime}$ ' was mutated to 5'-CCUUAGG-3'.

Luciferase reporter constructs. For the luciferase reporter constructs, DNA fragments encoding the chick and mouse FoxP1 3' UTR were amplified using the following primers: chick FoxP1 3' UTR, forward, 5' tatcggggaggggttaaaag- $3^{\prime}$ and reverse, $5^{\prime}$-acgatcccgaaacagaagtg- $3^{\prime}$; mouse FoxP1 3' UTR, forward, 5'-agtggacgacctttttgcac-3' and reverse, 5'ccagaggataaatgccagga- $3^{\prime}$. DNA fragments were subcloned into a pGL4.13 Luciferase vector (Promega).

As a control, chick Isl1 3' UTR was amplified using the following primers: forward, 5' -catccagagttttgatgttg-3'; reverse, $5^{\prime}$-ctattccaaacgcttatttagtg- $3^{\prime}$.

Endogenous miR-9 knockdown constructs. For endogenous miR-9 inhibition, the $22 \mathrm{nt}$ miR-9 binding site 1 in the chick FoxP1 3' UTR and $85 \mathrm{nt}$ of genomic sequences flanking the site 1 were amplified by PCR using the following primers: forward, 5'-gtcactcatttatttatgtag- $3^{\prime}$; reverse, $5^{\prime}$ ctcctttgatcaacaataagag- $3^{\prime}$. Two copies of the PCR product were subcloned into the pCAGIG vector. 


\section{A \\ Sequences for mature miR-9 and miR-9-Mut \\ miR-9: 5 '-UCUUUGGUUAUCUAGCUGUAUGA-3 miR-9-Mut: 5'-UCCUUAGGUAUCUAGCUGUAUGA-3}
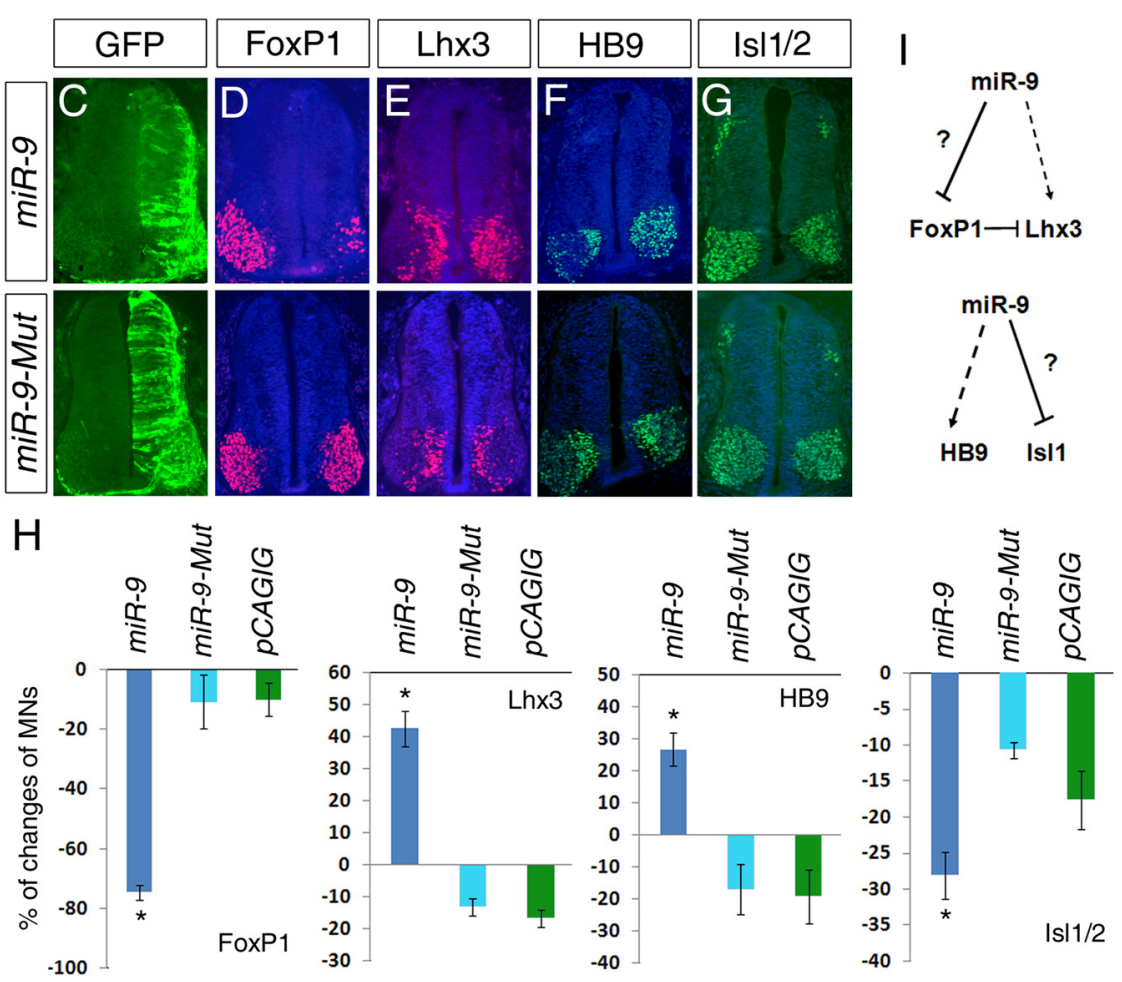

Figure 2. Overexpression of miR-9 alters motor neuron subtypes in the spinal cord. $A$, Sequences for mature miR-9 and the mutation of miR-9 (red) in the seed sequence that can abolish miR-9 activities. $\boldsymbol{B}, 0$ verexpression of miR- 9 as detected by in situ hybridization using anti-miR-9 probes. $\mathbf{C}-\mathbf{G}$, In brachial levels of stage 24 spinal cords, electroporated at stage 11 , overexpression of miR-9 but not miR-9 mutations (miR-9-Mut), detected by GFP expression, decreased MNs expressing FoxP1 and Is 11/2 but increased MNs expressing Lhx3 and HB9. $\boldsymbol{H}$, Quantification of MN numbers affected by miR-9 overexpression. $n>5$. FoxP1, ${ }^{*} p<$ 0.0001; LhX3, ${ }^{*} p<0.0001 ; \mathrm{HB} 9,{ }^{*} p<0.008 ;$ Is 11/2, ${ }^{*} p<0.001$. I, A prediction of miR-9 regulation: miR-9 may inhibit the expression of FoxP1 and/or Is|1.

In ovo electroporation. Hamburger and Hamilton (HH) stage 10-12 chick embryos were electroporated unilaterally (five 50-ms pulses at 25 V) with 1-3 $\mu \mathrm{g} / \mu \mathrm{l}$ DNA using an ECM830 electro-squareporator (BTX). Embryos were analyzed after 24, 48, and $96 \mathrm{~h}$ of incubation.

Tissue preparation and immunohistochemistry. Spinal cord tissues from the chick embryos were fixed in $4 \%$ paraformaldehyde (PFA) in PBS overnight, incubated in 25-30\% sucrose in PBS, embedded in OCT, and stored at $-80^{\circ} \mathrm{C}$ until use. The spinal cords were sectioned $(14-18 \mu \mathrm{m})$ using a cryostat.

For antigen recovery, sections were incubated in heated $\left(95-100^{\circ} \mathrm{C}\right)$ antigen recovery solution ( $1 \mathrm{~mm}$ EDTA and $5 \mathrm{~mm}$ Tris, $\mathrm{pH}$ 8.0) for 15-20 min and cooled down for 20-30 min. Before applying antibodies, sections were blocked in $10 \%$ normal goat serum in PBS with $0.1 \%$ Tween 20 for $1 \mathrm{~h}$. Sections were incubated with primary antibodies at $4^{\circ} \mathrm{C}$ overnight and visualized using goat anti-rabbit IgG Alexa Fluor 488, goat anti-mouse IgG Alexa Fluor 594, or anti-guinea pig IgG Alexa Fluor 594 (1:350; Invitrogen) for $1 \mathrm{~h}$ at room temperature. Images were captured using a Leica digital camera under a fluorescent microscope (Leica) or using a confocal microscope (Carl Zeiss).

Primary antibodies against the following antigens were used: Lhx3 [mouse, 1:20 (Developmental Studies Hybridoma Bank); rabbit, 1:100 (kindly provided by Dr. T. Jessell, Columbia University, New York, NY)], FoxP1 (1:100; kindly provided by Dr. B. Novitch, University of California, Los Angeles, Los Angeles, CA), bromodeoxyuridine (BrdU) (1:100; Invitrogen), GFP (1:500; Millipore Bioscience Research Reagents), Pax6

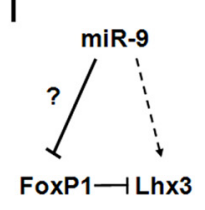

(1:30; Developmental Studies Hybridoma Bank), Neurofilament (NF) (1:20; Developmental Studies Hybridoma Bank), Lhx1 (1:20; Developmental Studies Hybridoma Bank), HB9 (1:20; Developmental Studies Hybridoma Bank), Isl1/2 [mouse, 1:20 (Developmental Studies Hybridoma Bank); rabbit, 1:100 (kindly provided by Dr. T. Jessell)], neuronal-specific nuclear protein (NeuN) (1:20; Developmental Studies Hybridoma Bank), Nkx6.1 (1:20; Developmental Studies Hybridoma Bank), and Chx10 (1:500; kindly provided by Dr. C. Cepko, Harvard Medical School, Boston, MA).

In situ hybridization. Digoxigenin (DIG)labeled sense and antisense mRNA probes were produced by in vitro transcription. The in situ hybridization on sections was performed as described previously (Sun et al., 2000). Briefly, the sections were hybridized at $65^{\circ} \mathrm{C}$ overnight and washed. After blocking for $2 \mathrm{~h}$, sections were labeled with anti-DIG antibody (1:1500; Roche) at $4^{\circ} \mathrm{C}$ overnight, washed, and stained with $\mathrm{BM}$ purple (Roche) at room temperature until ideal intensity. The images of in situ hybridization were collected using a Leica digital camera under a dissection scope (Leica).

In situ hybridization for microRNA detection was performed according to previously published methods with locked nucleic acid (LNA) probes (Obernosterer et al., 2007). The miR-9 LNA probes (Exiqon) were 3' end labeled with DIG-ddUTP with terminal transferase using the DIG-3' end labeling kit (Roche).

Western blotting analysis. Chick FoxP1 3' UTR was cloned into the pCMV-SPORT6FoxP1 image clone to create the expression vector pCMV-SPORT6-FoxP1-c-3' UTR. After $48 \mathrm{~h}$ cotransfection with pcDNA3-miR9-1, pcDNA3-miR-9-2, pcDNA3-miR-9Mut, pcDNA3-miR-17, or pcDNA3 vector in Neuro2a cells, $15 \mu \mathrm{g}$ protein extracts were fractionated by SDS-PAGE, immunoblotted, probed with anti-FoxP1 antibodies (1:500; kindly provided by Dr. B. Novitch), and subsequently probed with peroxidase-conjugated secondary antibodies (1:20,000; Sigma). Actin expression was detected by anti-Actin antibodies (1:200; Sigma). The signals were detected with Supersignal West Pico Chemiluminescent Substrate (Pierce).

Luciferase assays. Neuro-2a cells, grown in MEM supplemented with $10 \%$ fetal bovine serum, were transfected with pGL4.13-FoxP1-c-3' UTR and pcDNA3-miR-9-1, pcDNA3-miR-9-2, pcDNA3-miR-9Mut, pcDNA3-miR-17, or pcDNA3 vector itself, in a 1:2 ratio, using Lipofectamine (Invitrogen). After $48 \mathrm{~h}$, luciferase activity was measured and normalized to renilla luciferase activity.

Spinal cord motor neuron axonal tracing. HH stage 27-29 chick embryos were fixed in $4 \%$ PFA overnight at $4^{\circ} \mathrm{C}$. Coronal sections $(300 \mu \mathrm{m})$ of the spinal cord were collected using a vibratome. Single pieces of the fluorescent carbocyanide dye DiI tissue (NeuroVue Red; Molecular Targeting Technologies) were placed in the ventral horn and the medial region on the spinal cord sections. To allow the DiI to diffuse, spinal cord sections were kept in $4 \%$ PFA in the dark at $37^{\circ} \mathrm{C}$ for $3-5 \mathrm{~d}$. Images of DiI-labeled axons were captured using a Leica digital camera under a fluorescent microscope.

The thickness of motor neuron projections was measured using the NIH ImageJ software. Five or six points with an equal space along a projection from the root of the axon were selected for the measurement.

Quantification of FoxP1 levels. Nuclear FoxP1 levels were determined on sections using antibodies against FoxP1. Images were obtained by 

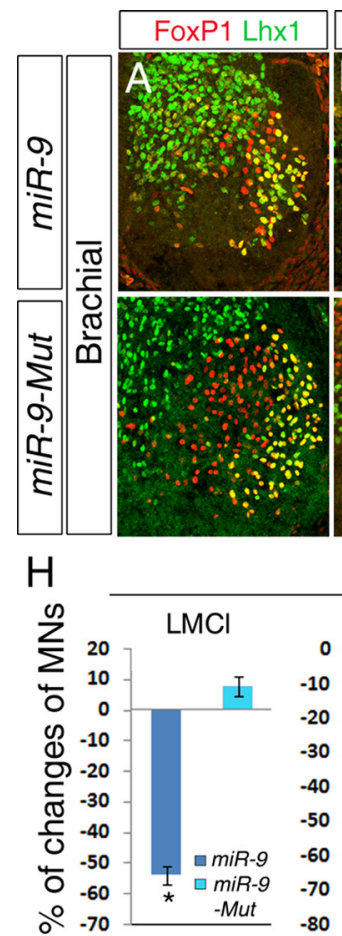

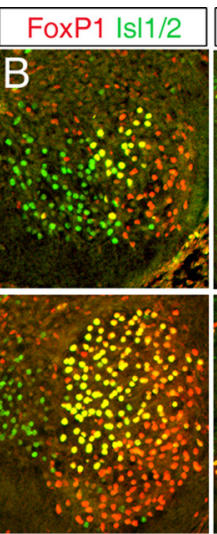

Brachial

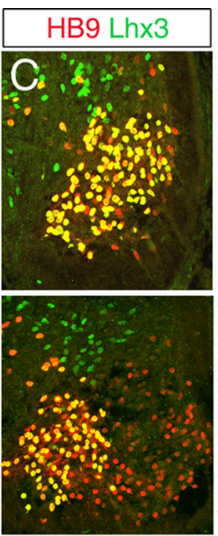

\begin{abstract}
(n)
\end{abstract}
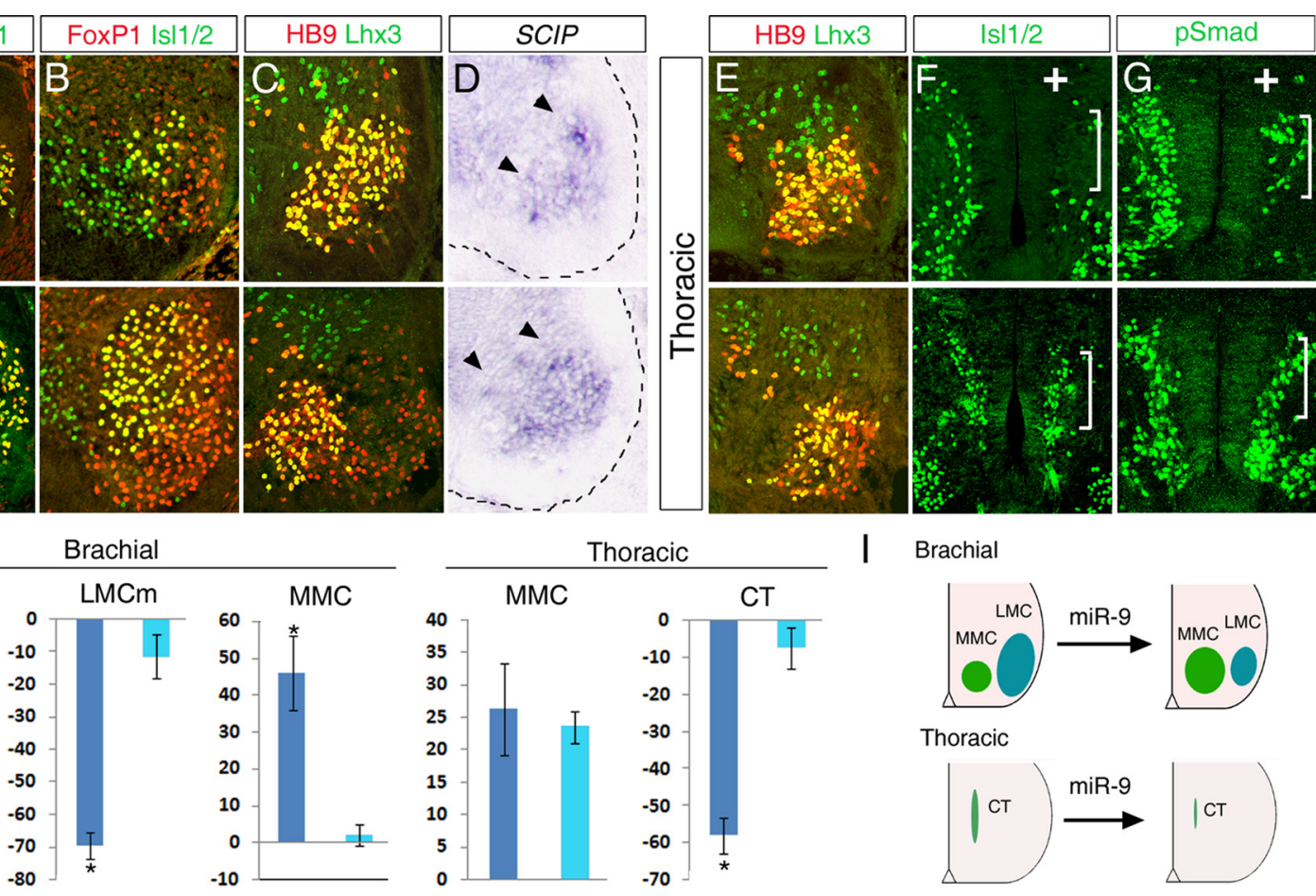

| Brachial

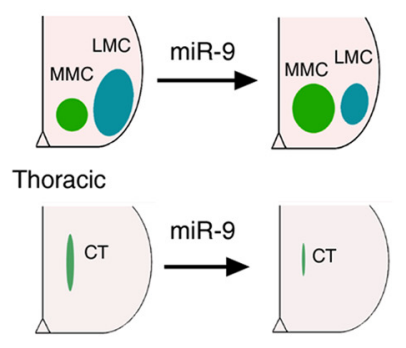

Figure 3. Overexpression of miR-9 alters columnar organization in the spinal cord. $\boldsymbol{A}-\boldsymbol{D}$, In brachial levels of stage 29 spinal cords, electroporated at stage 11 , overexpression of miR-9 but not miR-9 mutations (miR-9-Mut) decreased MNs in the LMCl (FoxP1 ${ }^{+} / \mathrm{Lhx}^{+}$) and LMCm (FoxP1 $\left.{ }^{+} / \mathrm{ss} 11 / 2^{+}\right)$but increased MNs in the MMC ( $\left.\mathrm{HBg}{ }^{+} / \mathrm{Lhx}^{+}\right)$. SCIP-expressing MNs in the LMC was also decreased (arrowheads). $\boldsymbol{E}-\boldsymbol{G}$, In thoracic levels of stage 29 spinal cords, electroporated at stage 11, overexpression of miR-9 decreased Isl1/2- and pSmad-expressing CT MNs (highlighted) but not MMC MNs. + indicates the electroporated side in the spinal cord. $\boldsymbol{H}$, Quantification of MN numbers in the LMC and MMC in brachial and thoracic levels in spinal cords electroporated with miR-9

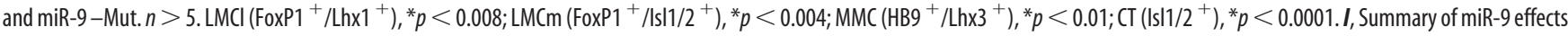
on MN columnar organization. High levels of miR- 9 inhibit MNs in the LMC and CT but promote MNs in the MMC in the brachial and thoracic levels.

fluorescence and confocal microscope using identical laser and gain configurations. All analyses were performed on sections on the same slide. Nuclear pixel intensity was determined using the NIH ImageJ software.

Motor neuron quantification. The total number of labeled motor neurons was quantified from $14-16 \mu \mathrm{m}$ sections collected from multiple embryos. The phenotype was determined by comparing the percentage of changes in total number of a specific motor neuron subtype between the electroporated and nonelectroporated side of the spinal cord. The Student's $t$ test was applied to determine the statistic significance between experimental and control groups.

Statistical analysis. At least five embryos electroporated with miR-9-1 or miR-9-2 or inhibition constructs and with miR-9-Mut or the PCAGIG empty vector were used for all statistical analyses. Data were shown as mean \pm SEM. Statistic comparison was made by ANOVA (unpaired $t$ test or ANOVA).

\section{Results}

miR-9 is transiently expressed in motor neurons in developing chick spinal cords

Recent studies and our own work have found that miR-9 is highly expressed in the cortex, the hindbrain, and the spinal cord, implying a potential role of miR-9 in the development of the CNS (Darnell et al., 2006; Leucht et al., 2008). In this study, we examined miR-9 expression patterns in chick spinal cords at different HH stages.

In brachial levels of stage 11 spinal cords, miR-9 expression was detected in the ventricular zone (VZ), which contains dividing cells, and in the floor plate (Fig. $1 \mathrm{~A}$ ). miR-9 was expressed in the VZ throughout the stages examined, with reduced expression at stage 29 (Fig. 1A-E). miR-9 expression in the ventrolateral spinal cord in which MNs reside was weak at stage 20 , increased by stage 24 , and undetectable by stage 29 , suggesting a transient expression in MN lineages (Fig. $1 B-D$ ).

To determine which motor neuron subtypes express miR-9, we compared miR-9 expression patterns with distinct MN markers in stage 24 spinal cords. The transcription factor FoxP1 is highly expressed in LMC MNs in the mouse and chick (Dasen et al., 2008; Rousso et al., 2008). In a superimposed image of adjacent sections of miR-9, detected by in situ hybridization, and FoxP1, detected by immunohistochemistry, miR-9 expression overlapped with most FoxP1-expressing (FoxP1 ${ }^{+}$) MNs (Fig. $1 F, G)$. Similarly, miR-9 was detected in Isl1/2-expressing domain (Fig. $1 H$ ). miR-9 expression overlapped with the low expression domain for HB9 and nonexpression region for Lhx3 but not with high Lhx3 and HB9 expression regions, in which MMC MNs reside (Fig. $1 \mathrm{I}, \mathrm{J}$ ). Our results indicate that miR-9 is mostly expressed in MNs of the LMC but not the MMC, implying a potential role of miR-9 for MN differentiation (Fig. $1 \mathrm{~K}$ ). Because miRNAs usually silence target gene expression, the overlapping expressions of miR-9, FoxP1, and Isl1/2 in LMC MNs lead us to examine whether miR-9 may negatively regulate FoxP1 and/or Isl1/2 expressions (Fig. $1 \mathrm{~K}$ ).

\section{miR-9 represses production of LMC MNs but promotes} differentiation of MMC MNs

To assess the function of miR-9 in MN specification, we examined the effects of miR-9 overexpression on MN development of the chick neural tube using in ovo electroporation at stage 10-11. We cloned two precursors for the chick miR-9, miR-9-1 and miR-9-2, which would be processed into indistinguishable mature miR-9 sequences, into a pCAGIG expression vector. To test 

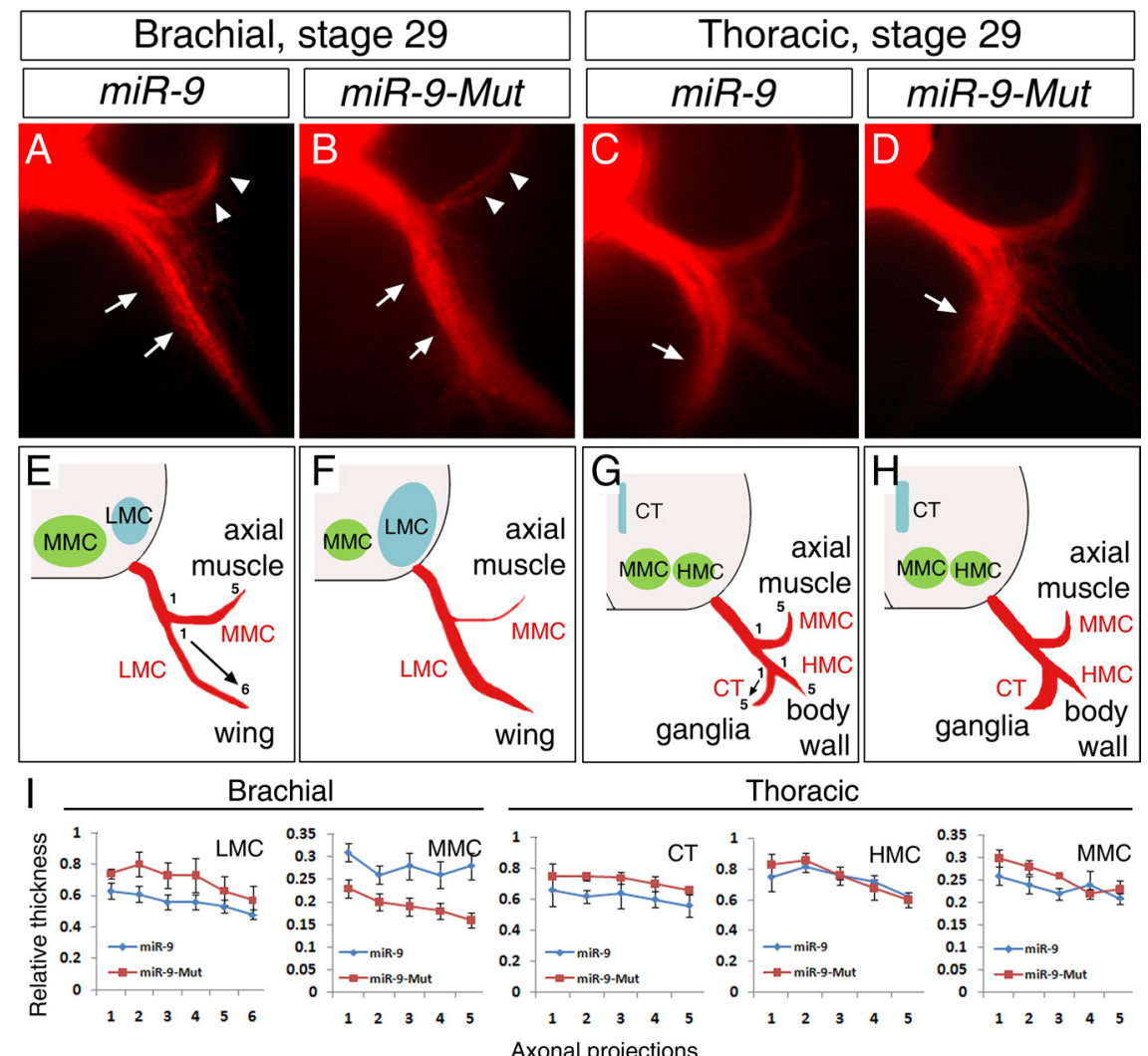

Thoracic

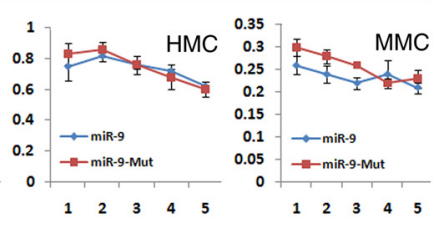

Figure 4. Overexpression of miR-9 alters motor neuron axonal projections. $\boldsymbol{A}, \boldsymbol{B}$, In brachial levels of stage 29 spinal cords, electroporated at stage 11 , overexpression of miR-9 but not miR-9 mutations (miR-9-Mut) reduced axonal fibers innervating the wing (arrows) but increased fibers projecting to the axial muscle (arrowheads). $\boldsymbol{C}, \boldsymbol{D}$, In thoracic levels of stage 29 spinal cords, electroporated at stage 11, overexpression of miR-9 but not miR-9 mutations (miR-9-Mut) reduced nerve fibers projecting to sympathetic ganglia (arrows). $\boldsymbol{E}-\boldsymbol{H}$, Schematic summaries of axonal projections altered by increased miR- 9 expression. I, Quantification of the relative thickness of axonal projections when miR-9 and miR-9 -Mut are overexpressed.

the specificity of miR-9 overexpression, we generated a miR-9-1 mutant construct (miR-9-Mut) with 3 nt substitutions in the seed sequence of miR-9, because the seed region of an miRNA has been demonstrated to be responsible for the strongest silencing activity (Bartel, 2009) (Fig. 2A). miR-9-Mut should be expressed as miR-9 but will lose miR-9 silencing ability on its target protein translation.

After expression of miR-9 at stage 11 and analysis at stage 24 when MN subtypes are specified, we detected ectopic miR-9 expression using in situ hybridization and the expression of the reporter gene GFP in the pCAGIG vector (Fig. $2 \mathrm{~B}, \mathrm{C}$ ). In brachial levels of the spinal cords, we found that the number of FoxP1 ${ }^{+}$ and Isl $1 / 2^{+}$neurons was reduced $70-75$ and $25-30 \%$, respectively, whereas the number of $\mathrm{Lhx}^{+}$and $\mathrm{HB} 9^{+}$neurons was increased 40 and 25\%, respectively (Fig. 2D-H) (supplemental Fig. 1, available at www.jneurosci.org as supplemental material). However, miR-9-Mut and the pCAGIG empty vector did not show significant alterations of MN subtypes. Similar effects of miR-9 on MN populations were also observed in thoracic and lumbar levels, suggesting a general role of miR-9 in altering specification of MN subtypes (supplemental Fig. 2, available at www. jneurosci.org as supplemental material) (data not shown). We also found that miR-9-1 and miR-9-2 precursors displayed a similar ability to alter the expression of MN markers, indicating that both precursors are processed similarly into identical mature miRNAs (supplemental Fig. 3, available at www.jneurosci.org as supplemental material). Therefore, we used miR-9-1 for our studies.
Because Lhx3 and HB9 are highly expressed in MMC MNs and FoxP1 and Isl1/2 are highly expressed in LMC MNs, increases of $\mathrm{Lhx}^{+}{ }^{+}$and $\mathrm{HB}^{+}{ }^{+} \mathrm{MN}$ numbers and decreases of FoxP1 ${ }^{+}$and Isl $1 / 2^{+}$ MNs caused by the overexpression of miR-9 indicate that miR-9 represses specification of the LMC MN subtype and promotes that of the MMC MNs. Because miRNAs function by silencing target mRNAs, the reduction in FoxP1 ${ }^{+}$and Isl1/2 ${ }^{+}$neurons suggests that miR-9 may directly or indirectly repress FoxP1 and Isl1/2 proteins (Fig. $2 I$ ). miRNA effects on promoting target gene expression are not yet well demonstrated (Vasudevan et al., 2007), and increased Lhx ${ }^{+}$MNs are likely a result of the repression of FoxP1, which usually inhibits Lhx3 expression (Dasen et al., 2008; Rousso et al., 2008) (Fig. 2I).

We next questioned whether altered MN subtypes are attributable to changes in $\mathrm{MN}$ progenitors. At both stages 20 and 24, dividing cells, labeled with a $30 \mathrm{~min}$ pulse of BrdU, and Pax6- and Nkx6.1expressing $\mathrm{MN}$ progenitors, showed no obvious changes when miR-9 was overexpressed (supplemental Fig. 4, available at www.jneurosci.org as supplemental material). Differentiated neurons, labeled with NeuN and NF, were not obviously affected, implying that the reduction of FoxP ${ }^{+}$and Isl1/2 ${ }^{+}$MNs was not caused by overall neurogenesis defects but $\mathrm{MN}$ subtype changes (supplemental Fig. 5, available at www. jneurosci.org as supplemental material). Loss of LMC MNs was not caused by cell death, because no obvious dead cells were detected in either LMC or MMC MNs (supplemental Fig. 6, available at www.jneurosci.org as supplemental material). Furthermore, interneuron precursors labeled by $\operatorname{Irx} 3$ and differentiated interneurons labeled by Chx10 were not altered, suggesting that miR-9 specifically affects MN differentiation (supplemental Fig. 5, available at www.jneurosci.org as supplemental material).

\section{Switches of MN columnar identities by miR-9}

To understand miR-9 functions in the assignment of MN columnar formation, we analyzed expression profiles of MN columnar markers in spinal cords overexpressing miR-9 and miR-9-Mut.

In brachial spinal cords at stage 29 , when MN columns are evident, the LMC is defined by FoxP1-, Isl1/2-, Lhx1-, and SCIP (suppressed cAMP-inducible POU)-expressing MNs (Kania and Jessell, 2003; Sockanathan et al., 2003; Dasen et al., 2008; Rousso et al., 2008), and the MMC is defined by Lhx3- and HB9expressing neurons (Sockanathan et al., 2003; Dasen et al., 2008; Rousso et al., 2008). We found a $>50 \%$ reduction of $\mathrm{FoxP}^{+} /$ $\mathrm{Lhx}^{+} \mathrm{MNs}$, which assemble the lateral LMC (LMCl), and $>65 \%$ reduction of FoxP1 ${ }^{+} / \mathrm{Isl} 1 / 2^{+} \mathrm{MNs}$, which form the medial LMC (LMCm), with overexpression of miR-9 but not miR9-Mut (Fig. $3 A, B, H$ ). Consistently, SCIP-expressing MNs in the LMC were also reduced (Fig. 3D). Furthermore, we found a $>45 \%$ increase of $\mathrm{Lhx}^{+} / \mathrm{HB}^{+}{ }^{+} \mathrm{MNs}$ in the MMC by overexpression of miR-9 (Fig. 3C,H). These results are consistent with 
A

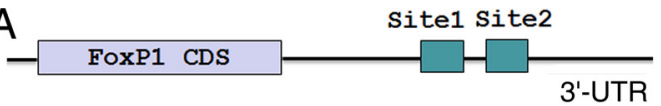

Site1

5' ... UUUUUUUUCCUAAAAUUACCAAAGA... | | | || ||

3. AgUAUGUCGAUCUAU-UGgUUUCU miR-9

Site2

5 ' . . CAAGUagauguCUgAaCcAAAgU. . .

| || || |

3. AGUAUGUCGAUCUAUUGGUUUCU miR-9

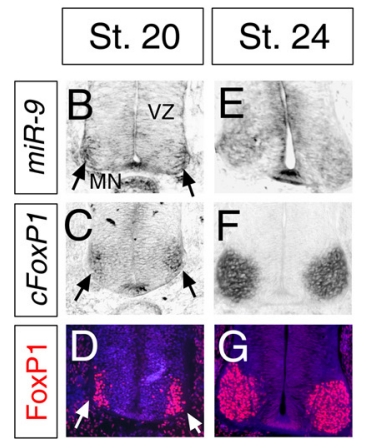

Co-expression of miR-9 and FoxP1 in LMC MNs

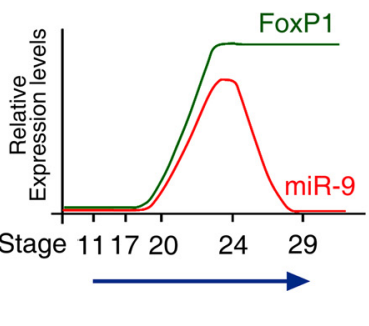

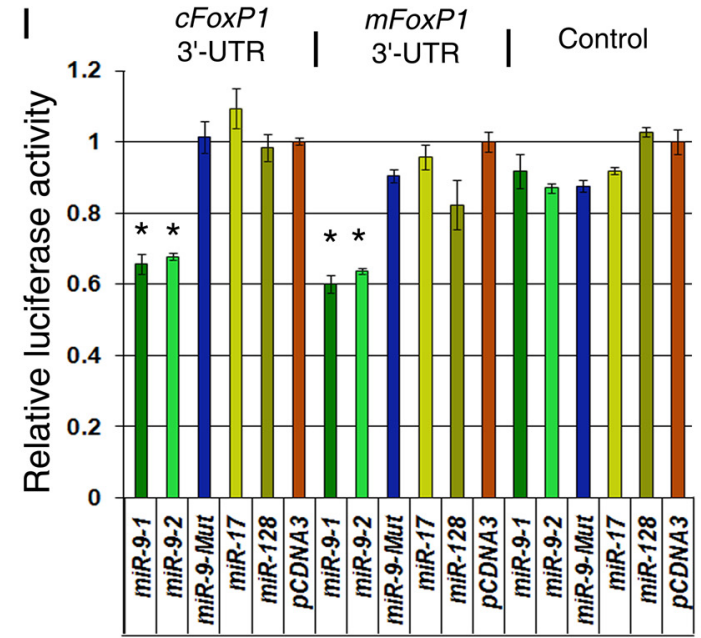

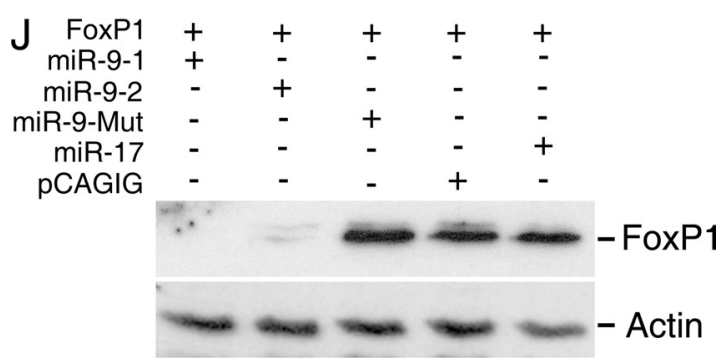

Figure 5. Transcription factor FoxP1 is a target for miR-9. A, There are two predicted targeting sites for miR-9 in the $3^{\prime}$ UTR of chick FoxP1. B, E, miR-9 was expressed in the VZ and MNs (arrows) in the LMC in stages (St.) 20 and 24 chick spinal cords. $\boldsymbol{C}, \boldsymbol{F}$, Chick FoxP1 (CFoxP1) mRNA was observed in the VZ and LMC MNs at stage 20 (C) but only in MNs at stage $24(\boldsymbol{F})$ as detected by in situ hybridization. D, G, FoxP1 protein was detected only in LMC MNs but not in the VZ in stages 20 and 24 spinal cords as detected by immunohistochemistry. $\boldsymbol{H}, A$ summary of miR-9 and FoxP1 coexpression in LMC MNs during development, with no expression at stages 11 and 17, and increased expression at stage 20 and 24. Although FoxP1 expression in LMC MNs is maintained, miR-9 expression in LMC MNs is greatly downregulated at stage 29. I, Luciferase assays of miR-9 targeting effects on the FoxP13' UTR. Both miR-9-1 and miR-9-2, but not miR-9-Mut and control miRNAs, miR-17, and miR-128, recognized the $3^{\prime}$ UTR sequences of $m$ FoxP1 (mouse) and $\left(F 0 x P 1\right.$ (chick) and resulted in reduced luciferase activities. $n=3$. cFoxP1: miR-9-1, ${ }^{*} p<0.0003 ;$ miR-9-2, ${ }^{*} p<$ 0.0001. mFoxP1: miR-9-1, ${ }^{*} p<0.0005$; miR-9-2, ${ }^{*} p<0.0003$.J, Both miR-9-1 and miR-9-2, but not miR-9-Mut and control miR-17, recognized the 3' UTR sequences of FoxP1 and knocked down FoxP1 protein levels as detected by Western blotting assays in Neuro2A cells. Actin was used as a loading control.

increased $\mathrm{Lhx}^{+}$and $\mathrm{HB}^{+}{ }^{+} \mathrm{MNs}$ and decreased Isl1/2 ${ }^{+} \mathrm{MNs}$ in stage 24 spinal cords (Fig. 2) (supplemental Fig. 6, available at www.jneurosci.org as supplemental material). Thus, miR-9 overexpression suppresses differentiation of LMC MNs, promotes differentiation of MMC MNs, and switches spinal MN columnar identities (Fig. 3I).

In thoracic spinal cords, the CT MNs, marked by Isl1/2 and pSmad (phosphorylated Sma- and Mad-related protein), were greatly reduced by miR-9 overexpression, whereas there were no significant changes of $\mathrm{HB}^{+} / \mathrm{Lhx}^{+} \mathrm{MNs}$ in the MMC when miR-9 and miR-9-Mut were overexpressed (Fig. 3E-I). Our results suggest that miR-9 selectively affects columnar organization in the chick spinal cord.

\section{Altering miR-9 expression changes $\mathrm{MN}$ axonal projections and $\mathrm{MN}$ pool differentiation}

Because miR-9 overexpression affects MMC and LMC MN differentiation, we examined whether $\mathrm{MN}$ axonal projections to target muscles have been altered. In stage 29 brachial spinal cords, DiI was placed into $\mathrm{MN}$ pools to trace MMC MNs, which innervate axial muscles, and LMC MNs, which innervate wing muscles in the chick. Axonal projections to the wing and axial muscle were detected, suggesting that miR-9 overexpression does not disrupt innervations of MNs to target muscles (Fig. $4 A, B$ ). However, comparisons in the thickness of nerve fibers indicated that miR-9 overexpression tended to cause reduced axonal projections to the wing but increased nerve fibers innervating axial muscles (Fig. 4).
Consistent with the expansion of MMC MNs, altered miR-9 expression may misroute some axons, which usually project to the wing, toward axial muscles normally targeted by MMC MNs.

In thoracic spinal cords, MMC MNs innervate axial muscles, HMC MNs innervate body wall muscles, and CT MNs innervate sympathetic ganglia (Sockanathan et al., 2003; Dasen et al., 2008; Rousso et al., 2008; Agalliu et al., 2009). By placing DiI in ventral and dorsomedial MN pools in stage 29 spinal cords, we found that miR-9 overexpression reduced axons projecting to the sympathetic ganglia but did not significantly affect nerve fibers toward the axial muscle and body wall (Fig. 4).

We next assessed whether LMC MN pool differentiation is affected by miR-9 overexpression. In stage 29 brachial spinal cords, Lhxl-expressing MNs in LMCl were reduced by miR-9 overexpression (Fig. 3A). The reduced medial LMC MNs, particularly labeled by Isl1/2, were replaced by $\mathrm{HB}^{+}{ }^{+} \mathrm{MNs}$, causing a displacement of $\mathrm{HB}^{+}{ }^{+} \mathrm{MNs}$ in $\mathrm{MN}$ pools (Fig. 3B) (supplemental Fig. 7, available at www.jneurosci.org as supplemental material). In addition, Nkx6.1 ${ }^{+} \mathrm{MNs}$ in the LMC were absent, and Cadherin 20 expression in LMC MNs and Raldh2 expression, particularly in LMCm MNs, were greatly reduced (supplemental Fig. 7, available at www.jneurosci.org as supplemental material). These results indicate that miR-9 overexpression affects LMC motor pool differentiation and identity.

Moreover, altered MN projections and motor pool identity, caused by miR-9 but not miR-9-Mut overexpression, recapitulate the defects in FoxP1 mutant spinal cords (Dasen et al., 2008; 
A

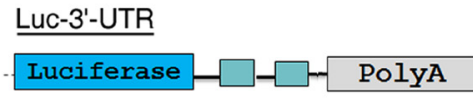

$\square$ FoxP1 3'-UTR binding site1 for miR-9

B

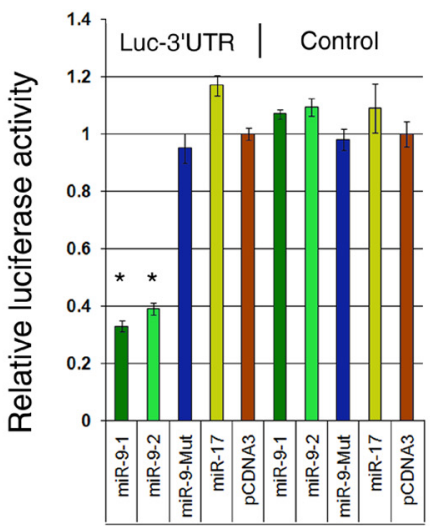

C

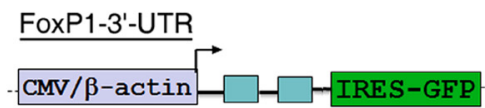

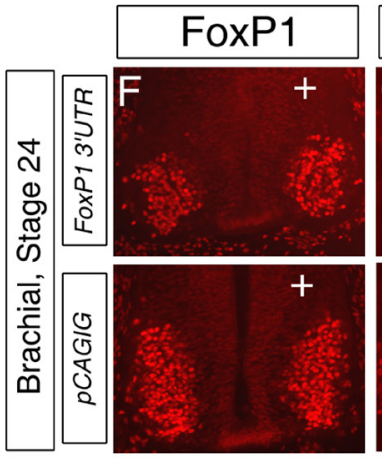

$\mathrm{J}$
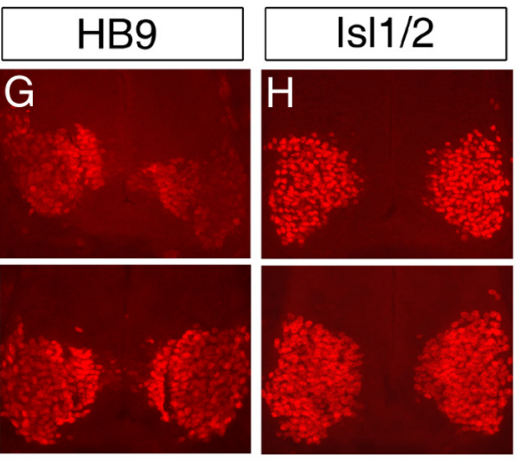

$\mathrm{N}$
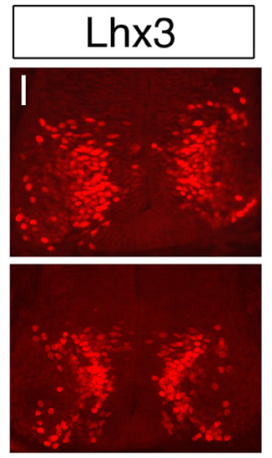



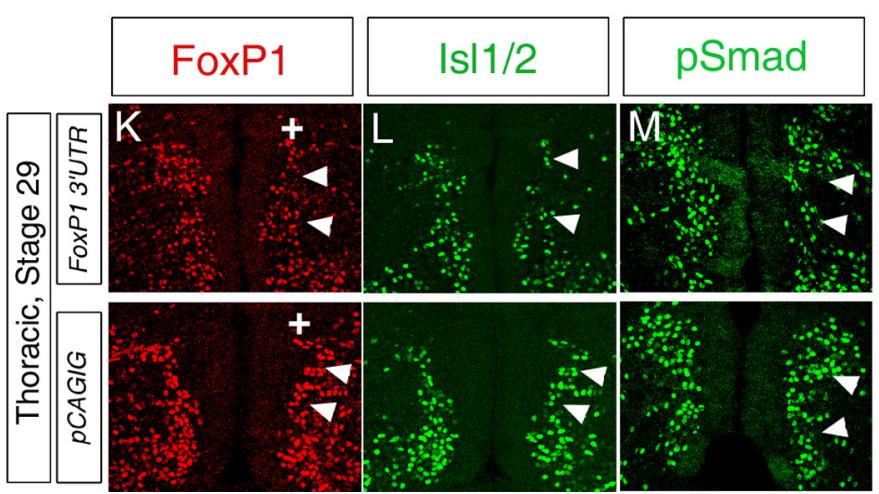

O

FoxP1-3'-UTR<smiles>[CH][CH]</smiles>
endogenous miR-9

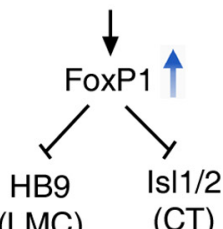

Figure 6. Overexpression of the $3^{\prime}$ UTR sequence of FoxP1 knocks down the endogenous miR-9. $A$, The binding site 1 for miR-9 in the $3^{\prime}$ UTR of chick FoxP1 was duplicated and cloned into a luciferase construct (Luc-3'UTR). B, Luciferase assays of miR-9 targeting effects on the FoxP1 3' UTR. Both miR-9-1 and miR-9-2, but not miR-9-Mut and the control miR-17, reduced luciferase activities. $n=3$. miR-9-1, ${ }^{*} p<0.004$; miR-9-2, ${ }^{*} p<0.0007$. C $-E$, Overexpression of the $3^{\prime}$ UTR sequence of chick FoxP1, electroporated at stage 11, knocked down endogenous miR-9 in MNs (arrows) in stage 24 (st. 24) spinal cords. F-J, Overexpression of the 3' UTR sequence of chick FoxP1 increased FoxP1 but decreased HB9 expression levels (intensity) in MNs in brachial levels of stage 24 spinal cords. $n>5$. FoxP1, ${ }^{*} p<0.0001 ;$ HB9, ${ }^{*} p<0.005$. Overexpression of the FoxP13' UTR did not change IsI1/2 and Lhx3 expression levels and patterns. + indicates the electroporated side in the spinal cord. $\boldsymbol{K}-\boldsymbol{N}$, In thoracic levels of stage 29 spinal cords, electroporated at stage 11, overexpression of the FoxP1 $3^{\prime}$ UTR, but not the $p C A G \mid G$ empty vector, decreased IsI1/2- and pSmad-expressing (T MNs (arrowheads). $n>7$. FoxP1, ${ }^{*} p<0.02 ;$ is $11 / 2,{ }^{*} p<0.01$. 0, Summary of miR-9 knockdown effects on MN columnar organization. Overexpression of the FoxP13' UTR sequences reduces endogenous miR-9 levels and in turn increases FoxP1 expression. Increased FoxP1 expression suppresses HB9 expression levels in LMC MNs in brachial levels of the spinal cord. In thoracic levels, increased FoxP1 inhibits Is11/2 expression in CT MNs and results in reduced CT MN numbers.

Rousso et al., 2008), suggesting that miR-9 likely functions through regulating FoxP1 expression levels (Figs. 2-4).

miR-9 affects MN columnar organization by a negative regulation of FoxP1 expression levels

miRNAs regulate target gene expression levels by affecting the stability of mRNAs and repressing target protein translation. Bioinformatic approaches predicted FoxP1 as a target of miR-9, which is consistent with the reduced FoxP1 levels when miR-9 was overexpressed (Figs. 2, 5A). We first compared expression patterns of miR-9 with FoxP1 mRNA and protein in developing chick spinal cords. At stages 11 and 17, miR-9 and FoxP1 were detected in the VZ. Expression of miR-9 overlapped with FoxP1 mRNA and protein in MNs by stage 20 and was increased by stage 24 (Figs. 1, 5B-G) (supplemental Fig. 8, available at www. jneurosci.org as supplemental material). At stage 29, whereas FoxP1 expression was maintained in LMC MNs, miR-9 expression was greatly reduced (Fig. $5 H$ ). These studies suggest that
miR-9 expression coordinates with FoxP1 in LMC MNs during the critical time of MN columnar formation.

We next examined whether miR-9 represses FoxP1 expression levels. The 3' UTR region of $c F o x P 1$ contains two miR-9 binding sites with identical seed sequences, which are essential for miRNA silencing effects (Fig. 5A). We cloned the entire 3' UTR sequences for both mouse and chick FoxP1 into a luciferase construct. Although control miRNAs (miR-17 and miR-128) had no effect on luciferase activity, miR-9-1 and miR-9-2, but not miR-9-Mut, significantly reduced luciferase activity of the constructs containing the 3' UTR for FoxP1, suggesting that miR-9 directly targets FoxP1 (Fig. 5I). To further confirm that miR-9 affects FoxP1 expression at a protein level, miR-9-1 and miR-9-2 were cotransfected with FoxP1 cDNA sequences containing both the coding region and the $3^{\prime}$ UTR in Neuro2A cells. miR-9-1 and miR-9-2, but not miR-9-Mut, significantly reduced FoxP1 protein but not FoxP1 mRNA (Fig. 5J) (data not shown). The overlapping expression of miR-9 and FoxP1 and the repressing function of 
miR-9 on FoxP1 suggest that miR-9 controls FoxP1 levels in the LMC MNs with a tuning regulation.

Because overexpression of miR-9 also reduces Isl 1 expression levels (Fig. 2G,I) and the 3' UTR region of Isll contains one miR-9 binding site (supplemental Fig. 9, available at www. jneurosci.org as supplemental material), we examined whether Isl1 is a potential target for miR-9. The luciferase assay revealed no effects of miR-9 on the Isl1 $3^{\prime}$ UTR, indicating FoxP1 as a specific target for miR-9 silencing (supplemental Fig. 9, available at www.jneurosci.org as supplemental material).

\section{Knocking down endogenous miR-9 expression increases FoxP1 levels and affects MN columnar differentiation}

To further examine whether FoxP1 level is regulated by miR-9 in vivo, miR-9 binding sequences in the FoxP1 3' UTR were overexpressed to knock down the endogenous miR-9. Duplicated site 1 binding sequences (200 bp each) for miR-9 in the cFoxP1 3' UTR were cloned into the luciferase construct (Fig. 6A). Both miR-9-1 and miR-9-2, but not miR-9-Mut and control miR-17, significantly decreased luciferase activities, which confirmed the direct targeting effect of miR-9 on FoxP1 3' UTR site 1 sequences (Fig. 6B).

Next, duplicated cFoxP1 3' UTR site 1 sequences were cloned into the pCAGIG vector for in ovo electroporation (Fig. 6C). We predicted that ectopic expression of miR-9 binding sequences from the FoxP1 3' UTR would reduce and saturate endogenous miR-9 activity and in turn diminish miR-9 functions. In the electroporated chick neural tube, we detected ectopic FoxP1 3' UTR expression and reduced miR-9 expression levels in the VZ and MNs in the ventral horn, indicating that ectopic expression of the FoxP1 3' UTR can be used to knock down endogenous miR-9 levels (Fig. 6D,E).

We then examined expression patterns and levels of MN markers when miR-9 was knocked down. In brachial levels of stage 24 spinal cords, endogenous miR-9 downregulation caused increased FoxP1 levels and decreased HB9 intensity but did not alter expression levels and patterns of Isl1/2 and Lhx3 (Fig. 6) (supplemental Fig. 8, available at www.jneurosci.org as supplemental material). Because miR-9 and FoxP1 are mostly coexpressed in LMC MNs, downregulation of miR-9 caused an increase of FoxP1 expression levels but did not alter FoxP1-expressing LMC MN numbers or cause cell death (Fig. 6) (supplemental Fig. 10, available at www.jneurosci.org as supplemental material). In thoracic levels, because CT MNs are FoxP1 dosage sensitive (Dasen et al., 2008; Rousso et al., 2008), increased FoxP1 expression, caused by miR-9 knockdown, reduced the number of CT MNs, as shown by decreased FoxP1-, Isl1/2-, and pSmadexpressing MNs (Fig. 6 $\mathrm{K}-\mathrm{O}$ ).

\section{Discussion}

Hox genes and their accessory proteins, such as FoxP1, are major determinants in motor neuron subtype specification, columnar organization, and axonal connections to targets. We found that microRNA-miR-9 plays an essential role in determining motor neuron subtypes and organizing spinal motor columns through a fine-tuning regulation of FoxP1 expression levels in motor neuron pools. Our results have revealed a new mechanism of motor neuron development that is mediated by microRNAs through a posttranscriptional gene expression regulation.

\section{miR-9 regulates motor neuron subtypes}

miR-9 displays dynamic expression patterns in developing chick spinal cords, with decreasing expression in the $\mathrm{VZ}$ and transient expression in MNs. miR-9 expression in the VZ, likely in progenitor cells, implies its function in progenitor development in the

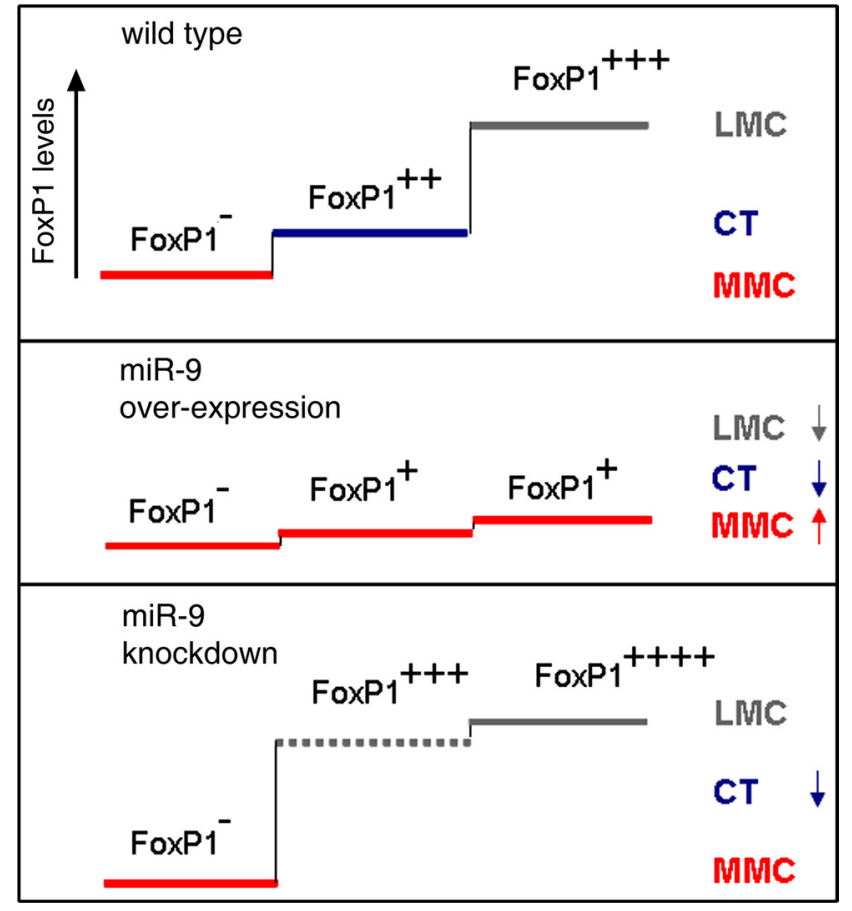

Figure 7. miR-9 modifies motor neuron subtypes and columnar organization in developing spinal cords by a tuning regulation of FoxP1 levels. FoxP1 levels are essential for the production of MNs in distinct columns, with high FoxP1 for MNs in the LMC, low FoxP1 for MNs in the CT, and no FoxP1 expression for MNs in the MMC. Overexpression of miR-9 suppresses FoxP1 levels and reduces MNs in the CT and switches LMC MNs into MMC MNs. Knockdown of miR-9 increases FoxP1 expression in thoracic levels and reduces CT MNs. Because miR-9 primarily overlaps with FoxP1-expressing cells only in the LMC in brachial levels, although knocking down miR-9 causes increased FoxP1 expressions, it does not change LMC MNs into other cell types. - indicates no FoxP1 expression, and numbers of + indicate the levels of FoxP1 expression in MNs.

neural tube. Previous studies have shown that miR-9 plays a role in neural stem cell differentiation and Cajal-Retzius cell production in developing brains (Shibata et al., 2008; Zhao et al., 2009). Our studies show that overexpression of miR-9 does not significantly affect $\mathrm{MN}$ and IN progenitors in the spinal cord. The effect of miR-9 in neurogenesis appears to be region and cell type specific in the CNS.

We have found that miR-9 expression in $\mathrm{MN}$ lineages is during the critical stages of MN subtype specification, from stage 20 to 24. In particular, miR-9 is positioned in MNs of presumptive LMC but not MMC. Unlike miR-124, which has a general effect on neurogenesis in the neural tube (Cao et al., 2007; Visvanathan et al., 2007; Yoo et al., 2009), our studies show that miR-9 overexpression promotes the generation of MMC MNs and represses LMC MNs but does not affect overall neurogenesis. Moreover, we found that miR-9 overexpression does not affect early production of oligodendrocyte precursors (data not shown). Thus, we have discovered a microRNA-miR-9 that regulates cell fate, particularly MN subtypes, in the neural tube.

miR-9 expression and the assembly of motor neuron columns and projections

Previous studies have demonstrated Hox genes and accessory factor FoxP1 as essential determinants in spinal MN columnar organization (Dasen et al., 2003, 2008; Rousso et al., 2008). Here, we find that miR-9 overexpression silences FoxP1 expression and reduces FoxP1 levels in LMC MNs, resulting in a switch of LMC MNs into MMC MNs (Fig. 7). Thus, in brachial levels of the spinal cord, miR-9 plays an important role in the assembly of 
LMC and MMC MN columns. In the signaling cascade, miR-9, perhaps in cooperation with Hox genes, regulates FoxP1 expression levels and defines MN columns (Dasen et al., 2008; Rousso et al., 2008; Jung et al., 2010). miR-9 may also function primarily during the early stages of MN columnar specification, because its expression is greatly downregulated in MNs by stage 29. Once MN columnar identity is established, miR-9 functions are no longer critical.

We also show that knocking down miR-9 increases FoxP1 levels but does not switch MN fates in the LMC and MMC in brachial spinal cords. First, miR-9 expression overlaps with most FoxP1-positive cells in LMC MNs. Knocking down miR-9 expression allows upregulation of its target FoxP1 only in LMC MNs. Increased FoxP1 in the LMC should not switch MN cell fates into other subtypes, such as MMC MNs (Dasen et al., 2008; Rousso et al., 2008) (Fig. 7). Second, although overexpression of FoxP1 3' UTR sequences greatly reduces endogenous miR-9, it does not completely abolish miR-9 expression and the low levels of miR-9 might continue to control target gene expression levels.

We have detected reduced CT MNs in thoracic spinal cords when miR-9 is either overexpressed or knocked down. Why does raised or lowered miR-9 level cause similar phenotypes in CT MNs? Previous work has shown that FoxP1 expression levels are critical for the generation of PGC MNs in mice (CT MNs in chick). CT MNs migrate dorsomedially and are sensitive to FoxP1 dosage, and low levels of FoxP1 expression favor the production of CT MNs (Dasen et al., 2008; Rousso et al., 2008) (Fig. 7). Overexpression or knockdown of miR-9 decreases or increases FoxP1 levels beyond the low or high thresholds of CT MN generation, respectively, and thus causes the reduction of CT MNs (Fig. 7).

Assembled MN columns project axons to their targets to form functional circuits. Consistent with observations in FoxP1 mutant mice (Dasen et al., 2008; Rousso et al., 2008), although miR-9 overexpression greatly reduces FoxP1 levels and LMC MN numbers, projections to the chick wing are still detectable. The environmental factors in the local targets such as wing muscles perhaps are sufficient to select proper axonal projections. Nevertheless, the trend of decreased nerve fibers to the wing and axons to the sympathetic ganglia, caused by increased miR-9 expression, indicates that miR-9 plays an important role in $\mathrm{MN}$ axonal innervations through the regulation of expression levels of molecules such as FoxP1.

\section{Fine-tuning regulation of miR-9}

miR-9 targeting effects on FoxP1 appear to be specific. We have shown that miR- 9 targets and silences FoxP1 by recognizing the 3' UTR of both chick and mouse FoxP1, indicating conserved miR-9 targeting effects. Mutating the miR-9 seed sequence relieves FoxP1 repression in either the spinal cord in vivo or cell lines as detected by luciferase and Western blotting assays. In addition, although Isl1/2-expressing cells are decreased when miR-9 is overexpressed and the 3' UTR of Isl 1 has a predicted binding site for miR-9, miR-9 does not show silencing effects on Isl1 expression. Decreased Isl1/2-expressing MNs are likely an indirect effect of reduced FoxP $1^{+}$cells.

Recent studies have shown that the tuning regulation of miRNAs in target gene expression is common in vertebrates (Shkumatava et al., 2009). The advantage of the tuning regulation is to ensure optimized protein outputs in cells through overlapping expression of miRNAs and their targets (Hobert, 2007; Karres et al., 2007; Biryukova et al., 2009).
miR-9-positive cells overlap with most, if not all, FoxP1expressing MNs in the LMC in chick spinal cords at stages 20 and 24. Why is the miR-9 tuning regulation of FoxP1 important in the differentiation of motor neuron pools? Previous studies have shown that the dosage of transcription factors, particularly FoxP1, is essential for specifying MNs in LMC, MMC, and PGC (Dasen et al., 2008; Rousso et al., 2008) (Fig. 7). In brachial spinal cords, the overlapping expression of miR-9 and FoxP1 and the targeting effect of miR-9 on FoxP1 expression may optimize FoxP1 levels in LMC MNs. Although higher amounts of miR-9 silence FoxP1 expression and reduce LMC MNs, lower levels of miR-9 permit increased FoxP1 expression in the LMC. In thoracic spinal cords, FoxP1 dosage is essential for generating PGC or CT MNs (Dasen et al., 2008; Rousso et al., 2008). Proper miR-9 expression levels perhaps ensure optimized FoxP1 dosage for the migration and production of CT MNs. Therefore, a balanced interaction between miR-9 and FoxP1, together with Hox gene regulations, is essential for proper output of FoxP1 protein levels in $\mathrm{MNs}$ and in turn defines spinal columnar organization and axonal connections to their targets (Dasen et al., 2008; Rousso et al., 2008) (Fig. 7).

Our studies reveal a fine-tuning regulation of FoxP1 expression levels by miR-9 during MN subtype specification and spinal columnar formation. The tuning regulation of miRNAs perhaps is a broadly applied mechanism for cell fate determination during development (Shkumatava et al., 2009). The technical advances in miRNA in vitro synthesis and delivery has made miRNAs a promising means for gene therapies. Revealing functions of miRNAs such as miR-9 in motor neuron specification may provide new methods of stem-cell-based therapies for spinal cord injuries.

\section{References}

Agalliu D, Takada S, Agalliu I, McMahon AP, Jessell TM (2009) Motor neurons with axial muscle projections specified by Wnt $4 / 5$ signaling. Neuron 61:708-720.

Bartel DP (2009) MicroRNAs: target recognition and regulatory functions. Cell 136:215-233.

Bartel DP, Chen CZ (2004) Micromanagers of gene expression: the potentially widespread influence of metazoan microRNAs. Nat Rev Genet 5:396-400.

Biryukova I, Asmar J, Abdesselem H, Heitzler P (2009) Drosophila mir-9a regulates wing development via fine-tuning expression of the LIM only factor, dLMO. Dev Biol 327:487-496.

Cao X, Pfaff SL, Gage FH (2007) A functional study of miR-124 in the developing neural tube. Genes Dev 21:531-536.

Carthew RW, Sontheimer EJ (2009) Origins and Mechanisms of miRNAs and siRNAs. Cell 136:642-655.

Cornbrooks EB, Newton CJ, Forehand CJ (1997) Development of differential preganglionic projections to pre- and paravertebral sympathetic ganglia. J Comp Neurol 382:1-18.

Darnell DK, Kaur S, Stanislaw S, Konieczka JH, Yatskievych TA, Antin PB (2006) MicroRNA expression during chick embryo development. Dev Dyn 235 [Erratum (2007) 236:333; Konieczka JK corrected to Konieczka $\mathrm{JH}]: 3156-3165$.

Dasen JS (2009) Transcriptional networks in the early development of sensory-motor circuits. Curr Top Dev Biol 87:119-148.

Dasen JS, Liu JP, Jessell TM (2003) Motor neuron columnar fate imposed by sequential phases of Hox-c activity. Nature 425:926-933.

Dasen JS, Tice BC, Brenner-Morton S, Jessell TM (2005) A Hox regulatory network establishes motor neuron pool identity and target-muscle connectivity. Cell 123:477-491.

Dasen JS, De Camilli A, Wang B, Tucker PW, Jessell TM (2008) Hox repertoires for motor neuron diversity and connectivity gated by a single accessory factor, FoxP1. Cell 134:304-316.

Fetcho JR (1992) The spinal motor system in early vertebrates and some of its evolutionary changes. Brain Behav Evol 40:82-97. 
Goulding M (2009) Circuits controlling vertebrate locomotion: moving in a new direction. Nat Rev Neurosci 10:507-518.

Gutman CR, Ajmera MK, Hollyday M (1993) Organization of motor pools supplying axial muscles in the chicken. Brain Res 609:129-136.

Hobert O (2007) miRNAs play a tune. Cell 131:22-24.

Hornstein E, Mansfield JH, Yekta S, Hu JK, Harfe BD, McManus MT, Baskerville S, Bartel DP, Tabin CJ (2005) The microRNA miR-196 acts upstream of Hoxb8 and Shh in limb development. Nature 438:671-674.

Jessell TM (2000) Neuronal specification in the spinal cord: inductive signals and transcriptional codes. Nat Rev Genet 1:20-29.

Jung H, Lacombe J, Mazzoni EO, Liem KF Jr, Grinstein J, Mahony S, Mukhopadhyay D, Gifford DK, Young RA, Anderson KV, Wichterle H, Dasen JS (2010) Global control of motor neuron topography mediated by the repressive actions of a single hox gene. Neuron 67:781-796.

Kania A, Jessell TM (2003) Topographic motor projections in the limb imposed by LIM homeodomain protein regulation of ephrin-A:EphA interactions. Neuron 38:581-596.

Karres JS, Hilgers V, Carrera I, Treisman J, Cohen SM (2007) The conserved microRNA miR-8 tunes atrophin levels to prevent neurodegeneration in Drosophila. Cell 131:136-145.

Kim VN, Han J, Siomi MC (2009) Biogenesis of small RNAs in animals. Nat Rev Mol Cell Biol 10:126-139.

Landmesser LT (2001) The acquisition of motoneuron subtype identity and motor circuit formation. Int J Dev Neurosci 19:175-182.

Lee RC, Feinbaum RL, Ambros V (1993) The C. elegans heterochronic gene lin-4 encodes small RNAs with antisense complementarity to lin-14. Cell 75:843-854.

Leucht C, Stigloher C, Wizenmann A, Klafke R, Folchert A, Bally-Cuif L (2008) MicroRNA-9 directs late organizer activity of the midbrainhindbrain boundary. Nat Neurosci 11:641-648.

Obernosterer G, Martinez J, Alenius M (2007) Locked nucleic acid-based in situ detection of microRNAs in mouse tissue sections. Nat Protoc 2:1508-1514.

Prasad A, Hollyday M (1991) Development and migration of avian sympathetic preganglionic neurons. J Comp Neurol 307:237-258.

Rousso DL, Gaber ZB, Wellik D, Morrisey EE, Novitch BG (2008) Coordinated actions of the forkhead protein Foxp1 and Hox proteins in the columnar organization of spinal motor neurons. Neuron 59:226-240.
Shah V, Drill E, Lance-Jones C (2004) Ectopic expression of Hoxd10 in thoracic spinal segments induces motoneurons with a lumbosacral molecular profile and axon projections to the limb. Dev Dyn 231:43-56.

Shibata M, Kurokawa D, Nakao H, Ohmura T, Aizawa S (2008) MicroRNA-9 modulates Cajal-Retzius cell differentiation by suppressing Foxg1 expression in mouse medial pallium. J Neurosci 28:10415-10421.

Shkumatava A, Stark A, Sive H, Bartel DP (2009) Coherent but overlapping expression of microRNAs and their targets during vertebrate development. Genes Dev 23:466-481.

Sockanathan S, Perlmann T, Jessell TM (2003) Retinoid receptor signaling in postmitotic motor neurons regulates rostrocaudal positional identity and axonal projection pattern. Neuron 40:97-111.

Stark A, Brennecke J, Bushati N, Russell RB, Cohen SM (2005) Animal MicroRNAs confer robustness to gene expression and have a significant impact on 3'UTR evolution. Cell 123:1133-1146.

Sun T, Jayatilake D, Afink GB, Ataliotis P, Nistér M, Richardson WD, Smith HK (2000) A human YAC transgene rescues craniofacial and neural tube development in PDGFRalpha knockout mice and uncovers a role for PDGFRalpha in prenatal lung growth. Development 127:4519-4529.

Vasudevan S, Tong Y, Steitz JA (2007) Switching from repression to activation: microRNAs can up-regulate translation. Science 318:1931-1934.

Visvanathan J, Lee S, Lee B, Lee JW, Lee SK (2007) The microRNA miR-124 antagonizes the anti-neural REST/SCP1 pathway during embryonic CNS development. Genes Dev 21:744-749.

Wightman B, Ha I, Ruvkun G (1993) Posttranscriptional regulation of the heterochronic gene lin-14 by lin- 4 mediates temporal pattern formation in C. elegans. Cell 75:855-862.

Wu Y, Wang G, Scott SA, Capecchi MR (2008) Hoxc10 and Hoxd10 regulate mouse columnar, divisional and motor pool identity of lumbar motoneurons. Development 135:171-182.

Yoo AS, Staahl BT, Chen L, Crabtree GR (2009) MicroRNA-mediated switching of chromatin-remodelling complexes in neural development. Nature 460:642-646.

Zhao C, Sun G, Li S, Shi Y (2009) A feedback regulatory loop involving microRNA-9 and nuclear receptor TLX in neural stem cell fate determination. Nat Struct Mol Biol 16:365-371. 